\title{
Patientensicherheit in der Physiotherapie
}

\section{Pia Fankhauser}

Vizepräsidentin physioswiss, Verantwortliche Bereich Kommunikation

Die Suche nach dem Wort «Patientensicherheit» ergab im KVG interessanterweise keinen Treffer. Es liegt also an den Leistungserbringern, diese mittels Qualitätssicherung zu garantieren. Die Aktionswoche der Stiftung Patientensicherheit Schweiz, in deren Stiftungsrat auch physioswiss, der Berufsverband der Schweizer Physiotherapeutinnen und Physiotherapeuten, vertreten ist, bietet Gelegenheit aufzuzeigen, welche Massnahmen die Physiotherapie zur Sicherung der Patientensicherheit ergreift.

Gemäss Art. 35 Abs. 2 lit. e des KVG gehören Physiotherapeuten und Physiotherapeutinnen zu den «Personen, die auf Anordnung oder im Auftrag eines Arztes oder einer Ärztin Leistungen erbringen». Der frühere Ausdruck «medizinische Hilfsperson» ist mittlerweile verschwunden. Aus Patientensicht gilt Art. 41 Abs. 1 KVG: «Die Versicherten können für die ambulante Behandlung unter den zugelassenen Leistungserbringern, die für die Behandlung ihrer Krankheit geeignet sind, frei wählen. Der Versicherer übernimmt die Kosten höchstens nach dem Tarif, der am Wohn- oder Arbeitsort der versicherten Person oder in deren Umgebung gilt.» physioswiss hat die Aufgabe, die Qualität der physiotherapeutischen Leistungen zu fördern, aber auch zu fordern. Dieser Qualitätsanspruch bedingt ein gemeinsames Verständnis der Gesundheitsberufe für das Mit-

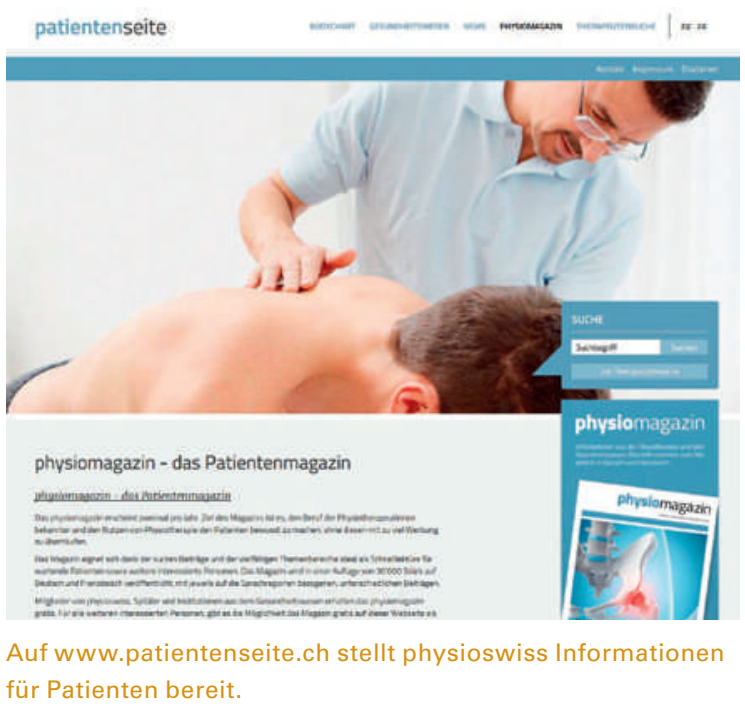

einander in der medizinischen Versorgung und für die gemeinsame Aufgabe der Qualitätssicherung. Die Grundsätze sind in der Berufsordnung von physioswiss festgehalten. Bereits in der Vernehmlassung zum Gesundheitsberufegesetz äusserte sich physioswiss wie folgt:

«Der Schaffung eines nationalen Berufsregisters räumt physioswiss höchste Priorität ein! Für die Patientensicherheit wie auch für die interdisziplinäre Zusammenarbeit der verschiedenen Berufsgruppen ist ein transparentes, jederzeit einsehbares Berufsregister für die nicht-universitären Gesundheitsberufe unerlässlich. Mit der stetig steigenden Mobilität der Akteure kann der Patientensicherheit und der interdisziplinären Zusammenarbeit nur Rechnung getragen werden, wenn schweizweit eine verlässliche Datenbank der Leistungsträger im Gesundheitswesen zur Verfügung steht.»

Für die Physiotherapie beginnt die Behandlung meist mit der Verordnung des Arztes, die als Kommunikationsmittel alle relevanten Angaben für eine zeitnahe und adäquate Behandlung beinhalten sollte. Dazu gehören neben der Diagnose und den Nebendiagnosen auch die wichtigsten Medikamente. Die physiotherapeutische Anamnese und Befunderhebung bildet die Grundlage für die Behandlung nach dem Prinzip des Clinical reasoning. Eine Zielvereinbarung mit einer Zeitachse ermöglicht dabei auf beiden Seiten die Qualitätskontrolle und weist frühzeitig auf Probleme hin. Die Behandlung wird mit einem Bericht an den verordnenden Arzt abgeschlossen.

Bei multimorbiden und chronisch kranken Menschen unabdingbar ist die Vernetzung der verschiedenen Behandelnden. Einerseits geht es also um die interprofessionelle Zusammenarbeit, andererseits aber auch um eine effiziente und korrekte Kommunikation.

Die modernen Technologien dazu sind vorhanden (Stichwort eHealth). Auch sie haben sich aber zu bewähren und müssen den Austausch von relevanten (!) Informationen erleichtern. Wir plädieren dafür, bei Einführung des elektronischen Patientendossiers auch die Funktion der Verordnung und die Berichterstattung zu 
überarbeiten. Für die in Art. 43 Abs. 6 KVG geforderte zweckmässige Versorgung, die qualitativ hochstehend zu erfolgen hat, ist dies elementar. Die IT ist in Folge ein wichtiger Teil der Patientensicherheit und beinhaltet auch den Datenschutz. Das elektronische Patientendossier erfordert ebenso die interprofessionelle Zusammenarbeit und eine klare Definition der für eine hohe Qualität nötigen Informationen. Deshalb beteiligt sich physioswiss auch im Rahmen der IPAG EPD (Interprofessionelle Arbeitsgruppe Elektronisches Patientendossier) an den Berichten zur eMedikation und zum elektronischen Austrittsbericht.

\section{Es geht um interprofessionelle Zusammen- arbeit, aber auch um eine effiziente und korrekte Kommunikation.}

Immer häufiger suchen Patienten direkt die Physiotherapie auf. Sei es, weil sie bereits einmal in Behandlung waren und sich in Zeiten des "Patient empowerment" selbständig für die ihnen passend scheinende Therapie entscheiden, oder sei es, weil sie Informationen erhalten haben, dass ihre Beschwerden dort behandelt werden können. Bei unspezifischen Rückenschmerzen gilt: Physiotherapie hilft sowohl unnötige Untersuchungen zu verhindern als auch eine rasche Arbeitsintegration zu ermöglichen. Vorläufig ist dies noch den Selbstzahlern vorbehalten, ein Prozess, der in Wandlung begriffen ist. Denn auch auf Seite der Physiotherapeutinnen und Physiotherapeuten entwickeln sich neue Rollen. Dies folgt der akademischen Entwicklung des Berufes. Der MSc-Studiengang hat sich etabliert. Der Verbandstitel Klinischer Spezialist / Klinische Spezialistin physioswiss kann in sechs Bereichen (Pädiatrie, Innere Organe und Gefässe, Neuromotorik und Sensorik, Muskuloskelettal, Geriatrie, Allgemeine Physiotherapie inkl. Psychomotorik) erlangt werden. Dies sichert die Weiterentwicklung des Berufes und damit auch der Qualität. Ebenso trägt die Forschung dazu bei, den Patienten die richtige Behandlung zukommen zu lassen.

Im Jahr 2009 wies das Swiss Medical Board darauf hin, dass bei Kreuzbandrissen die Physiotherapie die erste Wahl sein sollte und nur bei gravierenden Begleitverletzungen oder erfolgloser konservativer Behandlung eine Operation in Erwägung gezogen werden sollte. Diese Empfehlung wurde 2013 nach einer Review wiederholt [1]. Die Rollen in der medizinischen Versorgung sind diesen wissenschaftlichen Erkenntnissen anzupassen. Eine gute Ausbildung und die stetige Weiterbildung lässt dabei in der Praxis die «red flags» erkennen und garantiert damit die Qualität. Dafür setzen sich physioswiss und die Fachhochschulen gemeinsam ein.
Der Vernetzung mit anderen Berufsgruppen im Gesundheitswesen räumt physioswiss hohe Priorität ein. Ein Beispiel ist die Plattform Interprofessionalität (www.interprofessionalitaet.ch), die von den Hausärzten Schweiz lanciert wurde. Ein anderes Beispiel ist das Projekt «Sturzprävention» der bfu, wo diverse Broschüren interprofessionell erarbeitet wurden. Die qualitativ hochstehende integrierte Versorgung wird nur möglich, wenn die Berufsgrenzen nicht Mauern für die Patienten sind, sondern das Wissen um die Kompetenzen die Basis für einen professionellen fachlichen Austausch bildet. Dies gilt selbstverständlich sowohl für den stationären als auch den ambulanten Bereich.

Wie sollen die Patienten in einem sich dermassen dynamisch entwickelnden Gesundheitswesen aber die für sie relevanten Informationen erhalten? Wie kann Qualität sichtbar gemacht werden?

physioswiss stellt unter www.patientenseite.ch Informationen für (potentielle) Patienten bereit, verbunden mit einer Therapeutensuche für seine Mitglieder. Begleitet wird diese Seite vom physiomagazin, das in einer Auflage von 30000 Stück auf Deutsch und Französisch in Wartezimmer und Spitälern über Themen der Physiotherapie berichtet. Die aktuelle Ausgabe ist beispielsweise dem Schlaganfall und seinen Anzeichen gewidmet.

Für die Aktionswoche der Stiftung Patientensicherheit Schweiz nutzen wir unser Kommunikationsmittel physiofacts, das sich an Parlamentarier und Parlamen-

Wir plädieren dafür, bei Einführung des elektronischen Patientendossiers auch die Funktion der Verordnung und die Berichterstattung zu überarbeiten.

tarierinnen des nationalen Parlamentes richtet: Wir machen darauf aufmerksam, dass Behandlungen das Wort «Hand» beinhalten und spendieren ein Händedesinfektionsmittel. Dies im Bewusstsein, dass Patientensicherheit nur im Zusammenspiel aller beteiligten Berufsgruppen und deren Mitglieder erreicht werden kann. Wir erhoffen uns von politischer Seite auch dann Unterstützung, wenn es um finanzielle Aspekte geht. Eines ist klar: Auch in der Physiotherapie sind die berechtigten Ansprüche an hohe Qualität und Wissen nicht gratis zu haben. Patientensicherheit ist uns wichtig, sie sollte es allen sein.

Patientensicherheit ist eine interprofessionelle Aufgabe, die auf gute Kommunikation und Ausbildung angewiesen ist. Zum Billigtarif ist dies aber nicht zu haben.

1 Swiss Medical Board. Review Fachbericht «Ruptur des VKB: operative oder konservative Behandlung?». Zollikon: Swiss Medical Board; 2013. 